\title{
THREE-DIMENSIONAL LEE WAVES
}

\author{
by \\ T. Marthinsen \\ Institute of mathematics \\ University of Oslo
}

\begin{abstract}
Linear wave theory is used to find the stationary, trapped lee waves behind an isolated mountain. The lower atmosphere is approximated by a three-layer model with Brunt-Välsälä frequency and wind velocity constant in each layer. The Fourier-integrals are solved by a uniformly valid asymptotic expansion and also by numerical methods.

The wave pattern is found to be strongly dependent on the atmospheric stratification. The way the waves change when the parameters describing the atmosphere and the shape of the mountain vary, is studied. Further, the results predicted by the theory are compared with waves observed on satellite photographs. It is found that the observed wave patterns are described well by the linear theory, and there is good agreement between observed and computed wavelengths.
\end{abstract}




\section{$1 \quad$ Introduction}

Three-dimensional lee waves have recelved little study compared to the two-dimensional case. They are, however, often observed on satellite photographs, and four such observations were presented and analysed in a previous paper, Gjevik and Marthinsen (1978), referred to here as I. Some photographs taken by the Skylab crew have been presented by Fujita and Tecson (1977) and by Pitts et.al. (1977). A review of satellite observations of lee waves and vortex shedding has been given by Gjevik (1979).

It is clear from the investigations in I that the observed waves presented there are trapped lee waves, i.e. waves with no vertical propagation. A condition for such waves to exist is that the Scorer parameter, which is the ratio between the Brunt-Väisälä frequency and the wind velocity, decreases with height. Such situations have been studied by Scorer and Wilkinson (1956), Palm (1958), Sawyer (1962) and Crapper (1962). There is no emphasis in these works on comparing with observations, and the atmospheric models used do not apply to most cases where wave patterns are observed.

In I the atmosphere was approximated by a four layer model, and wave kinematics were employed to compare linear lee wave theory with the observations. The kinematic arguments give the phase lines which indicate possible appearances of the waves. A full solution, however, requires the calculation of the amplitudes, and that is the main purpose of the present paper.

In section 4 we study how the waves are effected by the stability 
profile and the shape of the mountain. The system of trapped lee waves behind an isolated mountain is composed of two parts, contained in a wedge, see fig. 3. Which of the two types will dominate the pattern is almost exclusively determined by the scorer parameter as a function of height. The wedge angle, denoted by $2 \alpha_{c}$, and the maximum amplitude vary rapidly when the stratification is changed, and it is possible to "tune" the atmosphere to situations where the wedge angle and the maximum amplitude and, accordingly, the wave energy take large values.

Some case studies follow in section 5. The atmospheric conditions and the shapes of the mountains corresponding to three of the observed cases in I, are approximated by a three-layer model and an obstacle with elliptical contours, and a comparison is made between observations and theory.

\section{The three layer model}

The air motion is described in a Cartesian coordinate system with the $z$-axis parallel to the mean wind, $U(z)$. The atmosphere in static equilibrium is assumed given by a three layer model, suggested by the observations in I, see fig. 1. N, the Brunt-Väisälä frequency, $\gamma=N / U$, the Scorer parameter, and $U$ are supposed to be piecewise constant. We scale all heights with $\mathrm{km}$ and wavenumbers and $\gamma$ with $\mathrm{km}^{-1}$. Typical values then are $\mathrm{h}_{0} \sim \mathrm{h}_{1} \sim 1, \gamma_{1} \sim 1, \gamma_{2} \sim 0.3,0.4$. The upper layer is extended to infinity because the more stable stratosphere has very little influence on lee waves in the lower troposphere, as shown for example by Ellassen and Palm (1961). 
Perturbing the velocity, pressure and density and writing the perturbation velocity and pressure as

$$
\begin{aligned}
(u, v, w) & =\frac{\bar{p}^{-\frac{1}{2}}(z)}{4 \pi^{2}} \int_{-\infty}^{\infty} \int_{-\infty}^{\infty}(\hat{u}, \hat{v}, \hat{w}) e^{-i(k x+l y)} d k d l \\
p & =\frac{\bar{p}^{\frac{1}{2}}(z)}{4 \pi^{2}} \int_{-\infty}^{\infty} \int_{-\infty}^{\infty} \hat{p} e^{-i(k x+l y)} d k d j
\end{aligned}
$$

where $\bar{p}(z)$ is the unperturbed density, we are, after making some standard approximations, led to the following equation for $\hat{w}$

$$
\frac{d^{2} \hat{w}}{d z^{2}}+\left(\frac{\gamma^{2}}{\cos ^{2} \varphi}-a^{2}\right) \hat{w}=0
$$

Here $a$ and $\varphi$ are defined by $a \cos \varphi=-k, a \sin \varphi=-1$. For the approximations made, see Crapper (1959).

We assume that the clouds making the lee waves visible, lie directly under the stable layer, that is, at $z=0$. The solution of (1) must fulfill the usual boundary conditions at $z=-h_{0}, 0, h_{1}$. When $z \rightarrow \infty$, we take the solution that goes to zero when $\gamma_{2}<|a \cos \varphi|$ and apply a radiation condition when $\gamma_{2}>|a \cos \varphi|$. We then obtain the elevation of the surface $z=0$, as

$$
n=\int_{-\pi}^{\pi} \int_{0}^{\infty} \frac{f(a, \varphi) T(a, \varphi)}{D(a,(p)} e^{i \psi} d a d \varphi
$$

where

$$
\begin{aligned}
T(a, \varphi) & =a \mu_{2} \sin \mu_{1} h_{1}+a \mu_{1} \cos \mu_{1} h_{1} \\
D(a, \varphi) & =\cosh a h_{0}\left(a \mu_{2} \sin \mu_{1} h_{1}+a \mu_{1} \cos \mu_{1} h_{1}\right) \\
& +\left(\frac{U_{1}}{U_{0}}\right)^{2} \cdot \operatorname{sinhah}{ }_{0}\left(\mu_{1} \mu_{2} \cos \mu_{1} h_{1}-\mu_{1} 2 \sin \mu_{1} h_{1}\right) \\
\psi & =a(x \cos \varphi+y \sin \varphi)=a r \cos (\varphi-\alpha)
\end{aligned}
$$


if $r, \alpha$ are polar coordinates in the $x y-p l a n e, x=r \cos \alpha$, $y=r \sin \alpha$. The function $f$ depends upon the shape of the mountain. When

$$
\gamma_{2}<|a \cos \varphi|<\gamma_{1}
$$

$\mu_{1}$ and $\mu_{2}$ are real and given by

$$
\begin{aligned}
& \mu_{1}=\left(\frac{\gamma_{1}^{2}}{\cos ^{2} \varphi}-a^{2}\right)^{\frac{1}{2}} \\
& \mu_{2}=\left(a^{2}-\frac{\gamma_{2}^{2}}{\cos ^{2} \varphi}\right)^{\frac{1}{2}}
\end{aligned}
$$

while if $|a \cos \varphi|<\gamma_{2}, \mu_{2}$ is the value in (6) multiplied by - I $\operatorname{sgn}(\cos \varphi)$, and if $|a \cos \varphi|>\gamma_{1}, \mu_{1}$ must be multiplied by $i$. We shall, however, only be concerned with wave components that satisfy the inequality (5). In this case, the equation

$$
D(a, \varphi)=0
$$

has real solutions, which correspond to trapped waves. These are postulated to lie downstream, and we obtain this expression for trapped waves

$$
n=2 \pi i \int_{-\pi / 2}^{\pi / 2} \frac{f(a, \varphi) T(a, \varphi)}{\frac{\partial}{\partial a} D(a, \varphi)} e^{i \psi} d \varphi+\begin{aligned}
& \text { complex } \\
& \text { conjugate }
\end{aligned}
$$

where $a=a(\varphi)$ is the solution of the dispersion relation, (7).

The function $a(\varphi)$ will take one of the two forms, $A$ or $B$, indicated in fig. 2. When the solution has the form $A$, both transverse and diverging waves will be trapped, in case $B$ the longest wave components, that is a part of the transverse waves towards the $\mathrm{x}$-axis 
will leak out. Phase lines illustrating both cases can be found in $I$.

There will also be higher modes solutions of (7), but computations show that the corresponding waves will have much smaller amplitudes and wedge angles than the highest mode for the parameter values that normally occur in the atmosphere.

\section{Integration methods}

To study trapped waves, we must evaluate the integral (8), and this can be done with asymptotic or numerical methods. The search for an asymptotic method starts with a study of the phase function, $\psi(\varphi)$. One finds that as long as $|\alpha|<\alpha_{c}$, the equation

$$
\frac{d \psi}{d \varphi}=0
$$

has two solutions, $\varphi_{1}$ and $\varphi_{2}$, that approach each other when $|\alpha| \rightarrow \alpha_{c}$, and fall together to a double root

$$
\frac{d \psi}{d \varphi}=\frac{d^{2} \psi}{d \varphi^{2}}=0
$$

for $\varphi=\varphi_{c}$ when $|\alpha|=\alpha_{c}$. When $|\alpha|>\alpha_{c}$, there are no solutions of $(9)$.

When $\varphi_{1}$ and $\varphi_{2}$ are not too close, one can use the method of stationary phase to obtain an approximate solution. From this method one finds that with $\left|\varphi_{1}\right|<\left|\varphi_{2}\right| \quad \varphi_{1}$ and $\varphi_{2}$ give points on the transverse and diverging waves respectively. Wave crests are phase lines, except when one comes out to $|\alpha|=\alpha_{c}$ where there is a 
shift of $\pi / 2$.

Phase lines corresponding to wave crests are shown in fig. 3 . The total wave field is the sum of the two wave types. The amplitudes of each of the two types increase as $|\alpha|$ grows from 0 to $\alpha_{c}$. When $\alpha=0$, the order of magnitude is $O\left(r^{-\frac{1}{2}}\right)$, while when $|\alpha|=\alpha_{c}$, it is $O\left(r^{-\frac{1}{3}}\right)$. The result is similar to the classical ship wave pattern, see for example stoker (1957).

The largest values of $n$ are expected to be found at the points $P$ where there is constructive interference. The method of stationary phase, however, does not apply when $\varphi_{1}$ is close to $\varphi_{2}$, and this is a great disadvantage because if the amplitudes are largest here, this would be the most interesting part to study. Chester, Friedman and Ursell (1957) and Ursell (1965) developed a general method applicable in this case, which gives an asymptotic expansion for (8), uniformly valid for all $\alpha$. In this method $\psi$ is represented by a cubic polinomial in a new variable $s$,

$$
\psi=\frac{1}{3} s^{3}-\beta^{2}(\alpha)+\nu(\alpha)
$$

To make (10) a regular (1-1) transformation

$$
\frac{d \varphi}{d s}=\frac{s^{2}-\beta^{2}}{\frac{d \psi}{d \varphi}}
$$

must be regular and nonzero. This requires $s\left(\varphi_{1}\right)=\beta, s\left(\varphi_{2}\right)=-\beta$, giving two equations to determine $\beta$ and $\nu$. The non-oscillating part of the integrand 1 s expanded in a series

$$
\sum_{n=0}^{\infty}\left\{p_{n}(\alpha)\left(s^{2}-\beta^{2}\right)^{n}+q_{n}(\alpha) s\left(s^{2}-\beta^{2}\right)^{n}\right\}
$$


To the lowest order the method gives an expression of the form

$$
\begin{aligned}
n & \approx P r^{-1 / 3} \sin \left(\frac{\left|\psi_{1}\right|+\left|\psi_{2}\right|}{2}\right) A 1\left(-\left[\frac{3}{4}\left(\left|\psi_{2}\right|-\left|\psi_{1}\right|\right)\right]^{2 / 3}\right) \\
& +Q r^{-2 / 3} \cos \left(\frac{\left|\psi_{1}\right|+\left|\psi_{2}\right|}{2}\right) A 1 \cdot\left(-\left[\frac{3}{4}\left(\left|\psi_{2}\right|-\left|\psi_{1}\right|\right)\right]^{2 / 3}\right)
\end{aligned}
$$

where $\varphi_{k}$ and $\psi_{k}=\psi\left(\varphi_{k}\right), k=1,2$ are complex when $|\alpha|>\alpha_{c}$. $P$ and $Q$ are combinations of the non-oscillating part of ( 8 ) with $\varphi$ taking the values $\varphi_{1}$ and $\varphi_{2}$, and they are independent of $r$ and a. A1 is an Airy-function and $A i^{\prime}$ its derivative. When $|\alpha|$ is less than and not too close to $\alpha_{c},\left|\psi_{2}\right|-\left|\psi_{1}\right|$ is large, and inserting the asymptotic expressions

$$
\begin{aligned}
& A i(-\xi) \approx \pi^{-\frac{1}{2}} \xi^{-1 / 4} \sin \left(\frac{2}{3} \xi^{3 / 2}+\frac{\pi}{4}\right) \\
& A i^{\prime}(-\xi) \approx \pi^{-\frac{1}{2}} \xi^{\frac{1}{4}} \cos \left(\frac{2}{3} \xi^{\frac{3}{2}}+\frac{\pi}{4}\right)
\end{aligned}
$$

(Abramowitz and Stegun (1965)) gives the stationary phase formula for $n$.

The formula (11) explains some qualitative features of the wave field, but to get maximum information out of it, it has to be evaluated numerically. It then turns out, however, that when $r$ is about 100 or less, it is more advantageous to evaluate (8) by ordinary Gauss quadrature, with the number of interpolation points varying between 48 and 64 . Evaluating the quadrature formulas takes less computer time, and the programming is much easier. Computations with both methods have, within the error bounds of the methods, given the same results. 


\section{Influence of mountain and stability}

The wave field depends on the stability parameters and the shape of the mountain. In order to study the effect of the mountain, we let the bottom topography be given by

$$
z=-h_{0}+5(x, y)
$$

and choose

$$
\zeta(x, y)=\zeta_{0} e^{-\left(\frac{x^{2}}{4 A^{2}}+\frac{y^{2}}{4 B^{2}}\right)}
$$

to specify the mountain. The mountain has elliptical contours, with the major axis along the $\mathrm{x}$-axis when $\mathrm{A}>\mathrm{B}$, and along the $\mathrm{y}$-axis when $B>A$. The function $f(a, \varphi)$ in (8) is then

$$
\begin{aligned}
& f(a, \varphi)=\zeta_{0} \frac{A B}{\pi} a e^{-a^{2}\left(A^{2} \cos ^{2} \varphi+B^{2} \sin ^{2} \varphi\right)} \\
& \text { Now } a=a(\varphi) \text {, so we define } \\
& \bar{f}(\varphi)=f(a(\varphi), \varphi)
\end{aligned}
$$

Fig. 4 shows $\bar{f}(\varphi)$ for one atmospheric stratification with different $A$ and $B$, but the same $\zeta_{0}$. Large function values for $\varphi<\varphi_{c}$ implies dominant transverse waves, and large values when $\varphi>\varphi_{c}$ means dominant diverging waves. In fig. 4 (i) and (ii) the mountain has the same shape. The major axis is in (i) across the wind, in (ii) along the wind. We see that $\bar{f}$ and hence the wave pattern is almost the same in these two cases.

Similar results are obtained with a different mountain in ( $v)$ and (vi). The wave pattern is almost unchanged, but the mountain with major axis along the wind generates smaller amplitudes.

(iii) and (iv) are both circular mountains, and the steeper the 
mountain, the more diverging waves will be seen.

A similar investigation has been made by Warren (1961) for surface waves behind a ship. He says: "Thus, for ships of broad beam moving at slow speeds, the transverse waves dominate the pattern, but for slender hlgh-speed craft the diverging waves are most prominent". Unfortunately he does not separate the effects of speed and form of the ship. Warren's statement is true, but from the present results we conclude that passing from low to high speed has a stronger influence on the wave pattern than passing from broad to slender objects.

In fig. 5 we see a part of the wave field behind the same circular mountain. The only difference between the two situations is the value of $\gamma_{1}$ which is 1.5 in the upper figure and 1.0 in the lower. If the Brunt-Väisälä frequency is the same, this means that the velocity in the lower figure is 1.5 times that in the upper. In both cases the phase lines go all the way into the $\mathrm{x}$-axis, making both wave systems possible. Notice also the different length scales on the figures.

We shall now study more systematically how the wave field depends on the stability parameters. We define a reference model by

$$
h_{0}=h_{1}=1.0, \quad r_{1}=1.0, \quad r_{2}=0.2, \quad \frac{U_{1}}{U_{0}}=1.0
$$

and vary $h_{0}, h_{1}, \gamma_{1}, \gamma_{2}$, one at a time. Define $\eta_{\text {MAX }}$ by

$$
\eta_{\text {MAX }}=\text { MAXIMUM } n \text { when } 100-\frac{\lambda}{2} \leq r \leq 100+\frac{\lambda}{2}
$$

where $\lambda$ is the wave length. Curves showing $\alpha_{c}$ and $\eta_{\text {MAX }}$ as functions of $\gamma_{1}$ are shown in fig. 6 a. A more detailed study of 
$\alpha_{c}$ 's dependence on $\gamma_{1}$ is given in $I$. In fig. $6 b-d$, the arguments are $\gamma_{2}, h_{0}, h_{1}$.

It is interesting to note that $\alpha_{c}$ and $\eta_{\operatorname{MAX}}$ reach their maxima for almost the same parameter values. Since the wave energy increases both when $\alpha_{c}$ and when $\eta_{\text {MAX }}$ increases, there must be small areas in the parameter space that give much more wave energy than outside these areas.

5. Comparison between theory and observations. Case studies

The preceding section showed that the wave pattern is quite sensitive to a change in the atmospheric stratification, and this complicates a comparison between theory and observation. The mountain shape seems, however, to be of somewhat less importance.

In I a four layer model was used to simulate the atmosphere, but a closer study has shown that an approximation by the simpler three layer model may be used instead. The results obtained by using the two methods are slightly different, but the differences are far less than the uncertainty induced by the choice of the actual parameter values. The cases we shall study and the choice of parameter are

\begin{tabular}{|lr|r|r|r|r|c|}
\multicolumn{1}{l|}{ Uase } & $\gamma_{1}$ & $\gamma_{2}$ & $h_{0}$ & $h_{1}$ & $\frac{u_{1}}{u_{0}}$ \\
\hline 1 - Jan Mayer & 1.Sept. 1976 & 0.5 & 0.2 & 0.9 & 1.7 & 1.1 \\
\hline 3 - Jan Mayen & 8.0ct. 1976 & 0.9 & 0.4 & 1.7 & 0.9 & 0.8 \\
\hline 4 - Bear Island 19. Sept.1976 & 1.5 & 0.5 & 0.7 & 0.8 & 1.0 \\
\hline
\end{tabular}


The cases 1,3 and 4 are the same as case I, III and IV in I, and the observations are contained there.

The phase lines for case 1 and case 4 are similar to those in I (I fig. 5), obtained by the four layer model. In case 3 omitting an upper layer allows longer waves to be trapped, and the phase lines include a part of the transverse waves. There is reason to believe on the basis of the present calculations and the previous reference to Eliassen and Palm (1961), that in this case the approximation used here is more correct than that used in $I$.

Case 1. Studying the satellite photograph, we observe that there are two diverging wave systems on the western side of the wake, indicating that each of the two peaks at Jan Mayen generates its own lee wave pattern. We therefore compute the sum of two wave systems, each of which is generated by a circular mountain. The line connecting the two tops, makes an angle of $45^{\circ}$ with the $x$-axis. In this computation the $\mathbf{z}$-axis is placed halfway between the mountains.

According to the theory, the amplitudes of the waves are proportional to the height of the mountain, but in the present case the mountain penetrates into the inversion layer, and this raises an objection against using linear wave theory. We do, however, obtain qualitatively good results with this method, and to make a choice, we take the taller mountain twice as high as the lower.

A part of the resultant wave field is shown in fig. 7 . The computation clearly shows that the observed wave system on the western 
side of the wake is composed of the waves from the two mountains.

Case 3. The wind direction in this case is from $E-N E$, so that the smaller peak at Jan Mayen lies at the lee side of Beerenberg. Also the inversion layer starts at the greater height than in case 1. These features may explain the fact that there is only une wave system to be seen on the photograph, and we use a circular mountain to simulate Beerenberg. There is symmetry about the $\mathrm{x}$-axis, and a part of the wave field with $\mathrm{y}>0$ is shown in fig. 8 a.

Case 4. The mountain used here has elliptical contours with $A=1.8, B=3.0$. This is a rough approximation to the southeastern part which is a plateau, taller than the rest of Bear Island. It is difficult to determine from the sateli1te photograph whether the transverse or the diverging wave system is observed in this case, but the computation which is shown in fig. $8 \mathrm{~b}$ leads to transverse waves only. The observed deformation of the wave field is probably due to a horizontal variation in the wind velocity. The wavelength in the center of the wake is measured to 9 on the photograph while the computation gives 10.2. This is considered good agreement. There is also good agreement between observed and computed wavelengths in case 1 and 3 .

\section{Conclusive remarks}

We conclude from the preceding results that although a simple calculation of phase lines explains some important features of the observed wave forms, finding the displacement heights in the waves is 
necessary if one wants to compare calculations with observations. The measured distance between two wave crests is not necessarily the wavelength, because in cases when both transverse and diverging waves exist, the observed wave is the sum of the two wave types which have different wavelengths. It is found in the cases examined that there Is good agreement between linear lee wave theory and observations as concerns the form of the wave pattern, for example the distance between wave crests. One cannot, however, deduce from satellite photographs the size of the amplitudes or the vertical shape of the waves, making it impossible to draw conclusions with respect to these characteristics.

The wave pattern is very sensitive to variations in the atmospheric stratification. This complicates a comparison between theory and observation, as it is difficult to know exactly what parameter values to use in the specific situations. One does not, however, need to be that cautious in specifying the mountain. A moderate change in the mountain parameters does not lead to great changes in the wave pattern,

\section{Acknowledgement}

The author's thanks are due to professor B. Gjevik, who suggested the problem and offered much advice during the research and the writing of the manuscript. 


\section{REFERENCES}

Abramowitz, M. and Stegun, I.A. 1965

Handbook of mathematical functions, Dover, New York.

Chester, C., Friedman, B. and Ursell, F. 1957

An extension of the method of steepest descent, Proc. Camb. Phil. Soc., 53, 599-611.

Crapper, G. D. 1959 A three-dimensional solution for waves in the lee of mountains, J.Fluid Mech., 6 , 51-76.

Crapper, G. D. 1962 Waves in the lee of mountains with elliptical contours, Phil. Trans. R. Soc., London, Series A 254, 601-624.

Eliassen, A. and Palm, E. 1961

On the transfer of energy in stationary mountain waves, Geof.Publ. (OsIo), XXII, No.3, 1-23.

Fufita, T.T. and Tecson, J.J. 1977

Mesoscale wake clouds in Skylab photographs, Skylab explores the earth, NASA SP-380,463-477.

Gjevik, B. 1979 Orographic effects revealed by satellite pictures: Mesoscale flow phenomena, Orographic effects in planetary flows, GARP Publication, Ch. 9, (To be published).

Gjevik, B. and Marthinsen, T. 1978

Three-dimensional lee-wave pattern, Quart. J.R. Met. Soc., 104, 947-957. 
Palm, E. 1958 Two-dimensional and three-dimensional mountain waves, Geof.Publ. (OsIo), XX, No. 3, 1-25.

Pitts, D., Lee, J.T., Fein, J., Sasaki, Y. Wagner, K. and Johnson, R. 1977

Mesoscale cloud features observed from skylab, Skylab explores the earth. NASA SP-380,479-501.

Sawyer, J.S. 1962 Gravity waves in the atmosphere as a three dimensional problem, Quart. J. R. Met. Soc., 88, 412-425.

Scorer, R.S. and Wilkinson, M. 1956

Waves in the lee of an isolated hill, Quart. J.R. Met. Soc., 82, 419-427.

Stoker, J.J. 1957 Water waves, Interscience, New York.

Ursell, F. 1965 Integrals with a large parameter. The continuation of uniformly asymptotic expansion, Proc. Camb. Phil. Soc., 61, 113-128.

Warren, F. W. G. 1961

A stationary phase approximation to the ship-wave pattern, J.Fluid Mech., 10, 584-592. 


\section{Figure captions}

Figure 1 The atmospheric model: Scorer parameter, $\gamma$, and mean wind velocity, $U$, as functions of height, $z$.

Figure 2 Solutions of dispersion relation. Case A: Transverse waves fully developed. Case B: Part of transverse waves leak out. Upper and lower curves are $\gamma_{1} / \cos \varphi$ and $\gamma_{2} / \cos \varphi$ respectively, bounding the region of trapped lee waves. There is symmetry about the a-axis.

Figure 3 Phase lines corresponding to wave crests for $y>0$. Half wedge angle $\alpha_{c}$. There is symmetry about the $x$-axis.

Figure 4 The function $\bar{f}(\varphi)$ defined in (12) for one atmospheric stratification with different mountains. $\varphi$ is measured in radians, $A, B$ in $\mathrm{km}, \bar{f}$ in $\mathrm{km}^{2}$ when $\zeta_{0}=1 \mathrm{~km}$.

Figure 5 Lines of constant, positive values of the elevation, $n$. There is symmetry about the $\mathrm{x}$-axis.

5 a : $r_{1}=1.5 \mathrm{~km}^{-1}$.

$5 \mathrm{~b}: r_{1}=1.0 \mathrm{~km}^{-1}$.

The values of $\gamma_{2}, h_{0}, h_{1}, U_{1} / U_{0}$ are respectively $0.2 \mathrm{~km}^{-1}$, $1.0 \mathrm{~km}, 1.0 \mathrm{~km}, 1.0$.

Figure 6 Maximum amplitude, $\eta_{\text {MAX, and half wedge angle as functions }}$ of $r_{1}, r_{2}, h_{0}, h_{1}$. 
Figure 7 Lines of constant, positive $n$ in case 1. The coordinates of the mountain tops are: Beerenberg $\mathrm{x}=-10 \mathrm{~km}, \mathrm{y}=10 \mathrm{~km}$. Smaller mountain $\mathrm{x}=10 \mathrm{~km}$, $y=-10 \mathrm{~km}$. The marks show the $\mathrm{y}$-coordinates of the mountains.

Figure 8 Lines of constant, positive $n$ in 8 a: case 3 , 8b: case 4. There is symmetry about the $\mathrm{x}$-axis. 


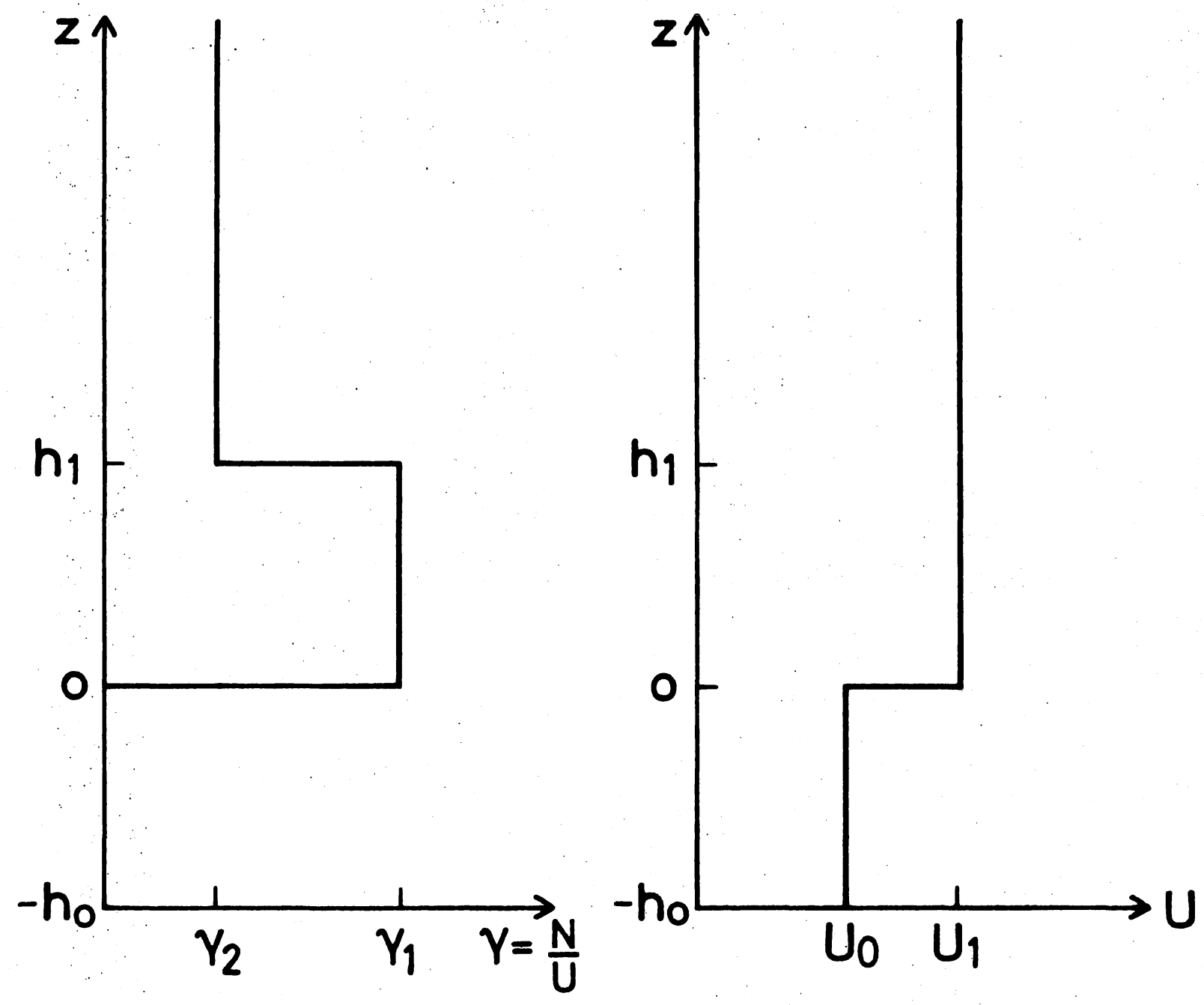

Fig. 1 


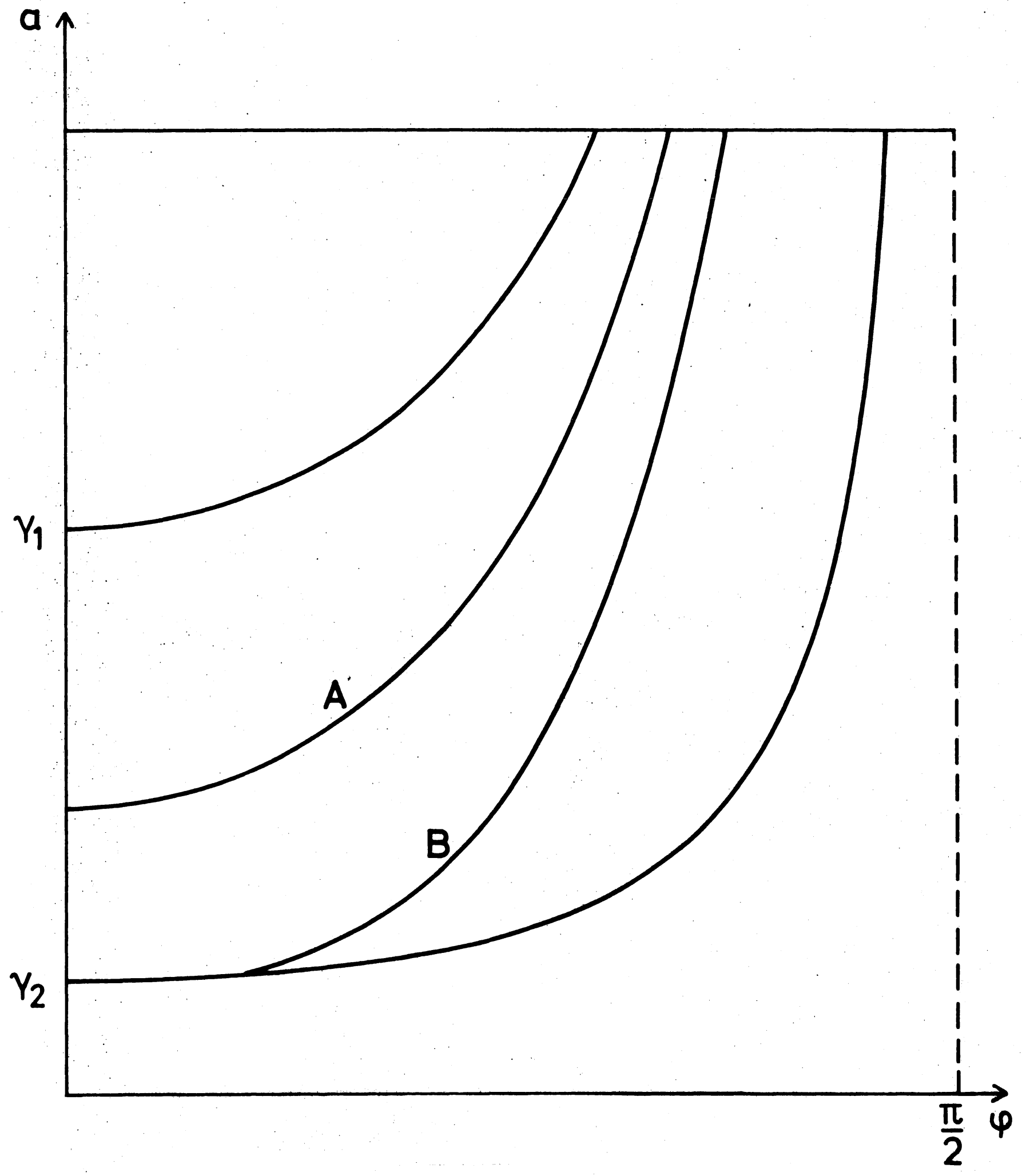

Fig. 2 


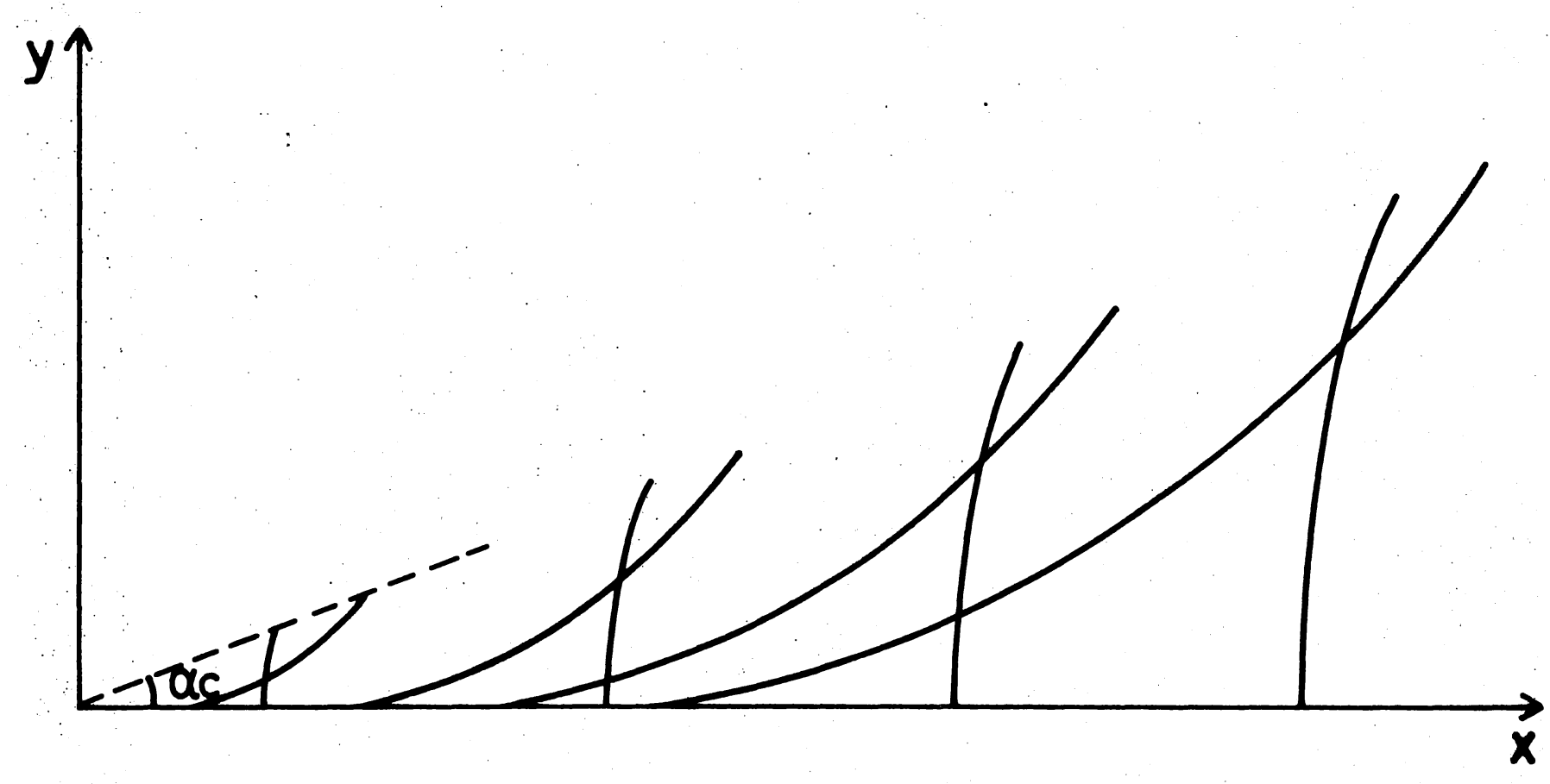

Fig. 3 


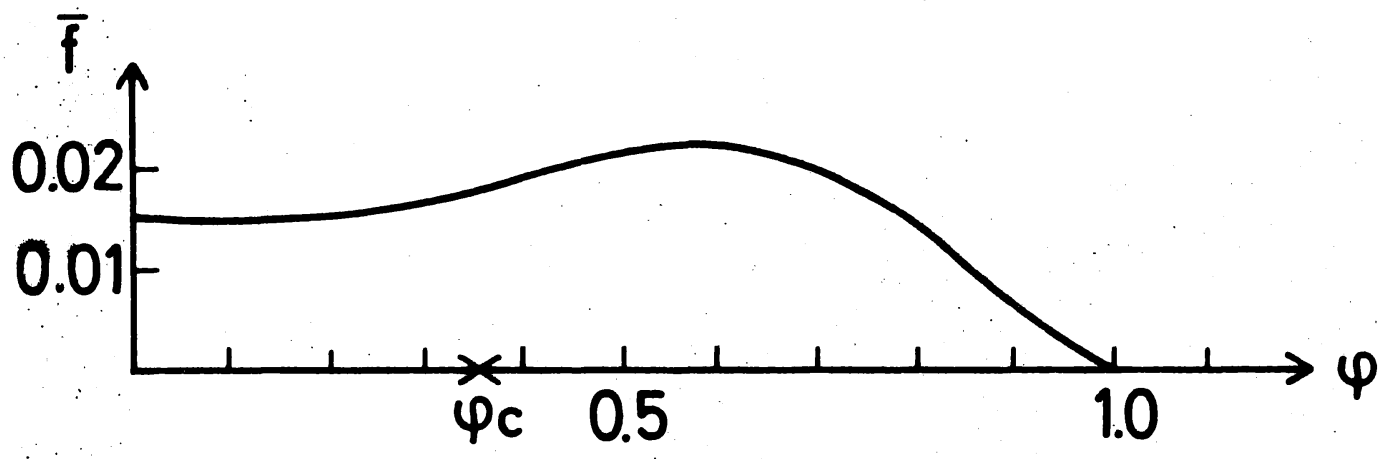

(i)

$A=0.1$

$B=1.0$

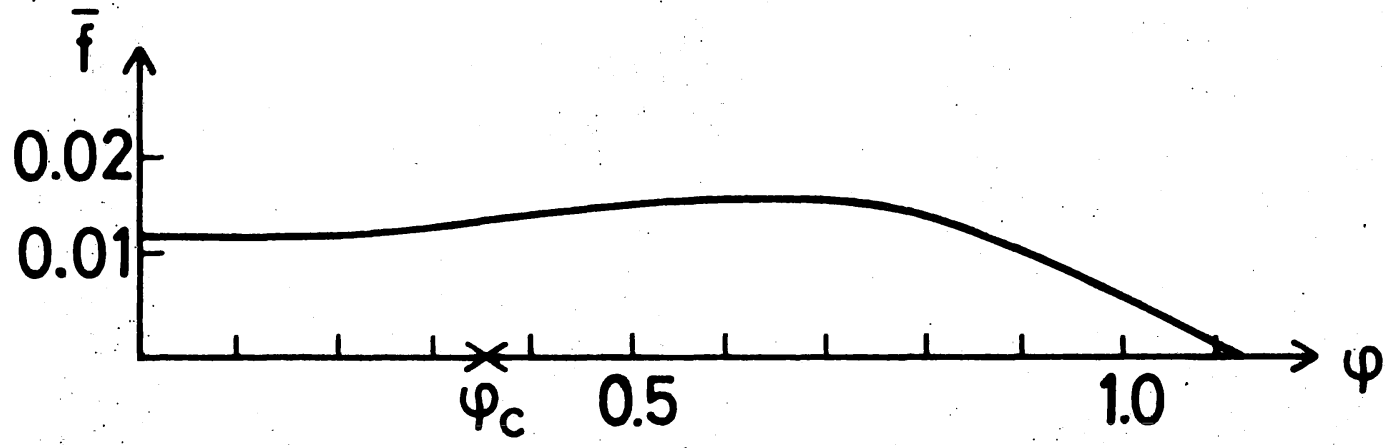

(ii)

$A=1.0$

$B=0.1$

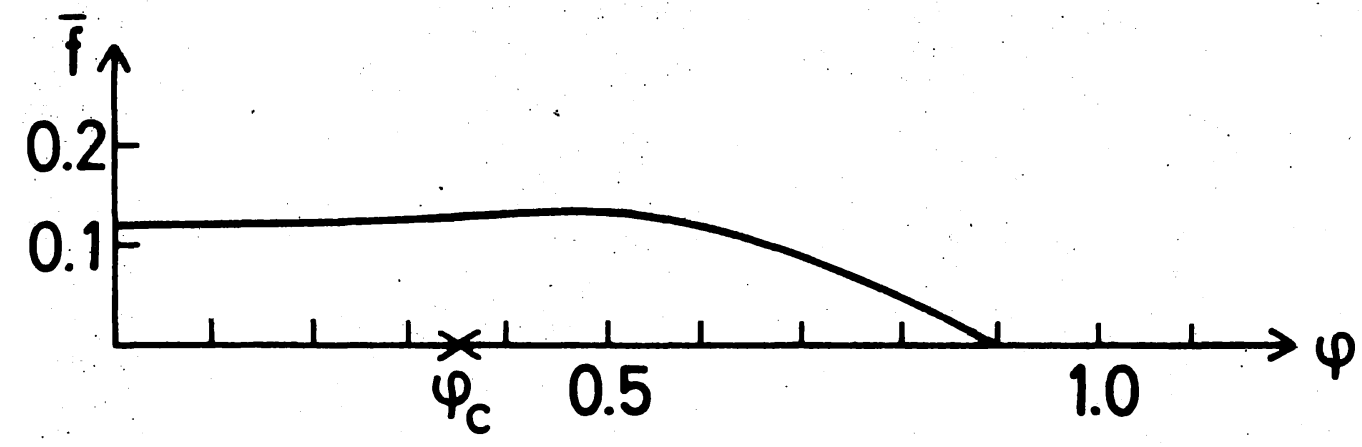

(iii)

$A=1.0$

$B=1.0$

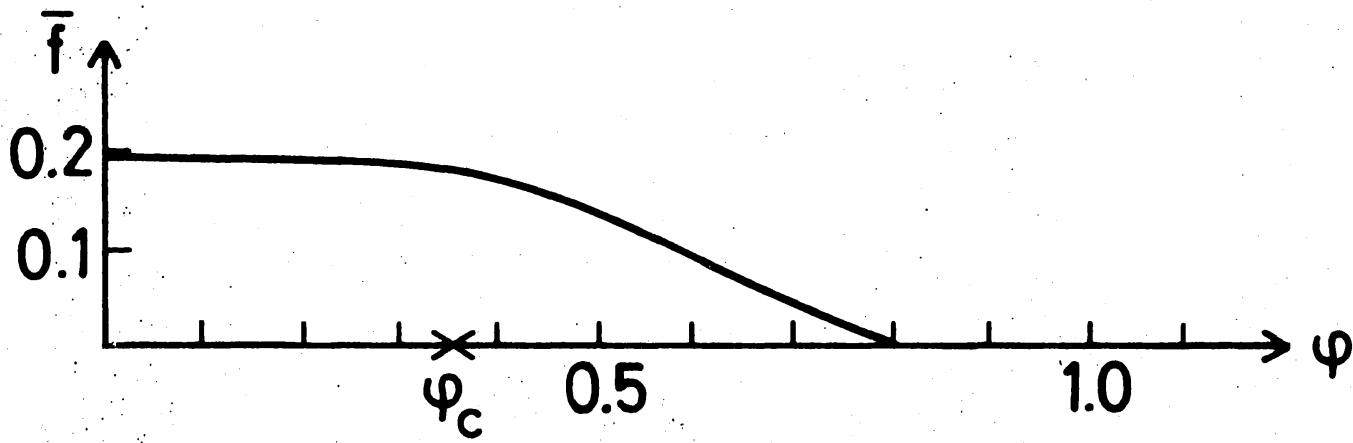

(iv)

$A=\sqrt{2}$

$B=\sqrt{2}$

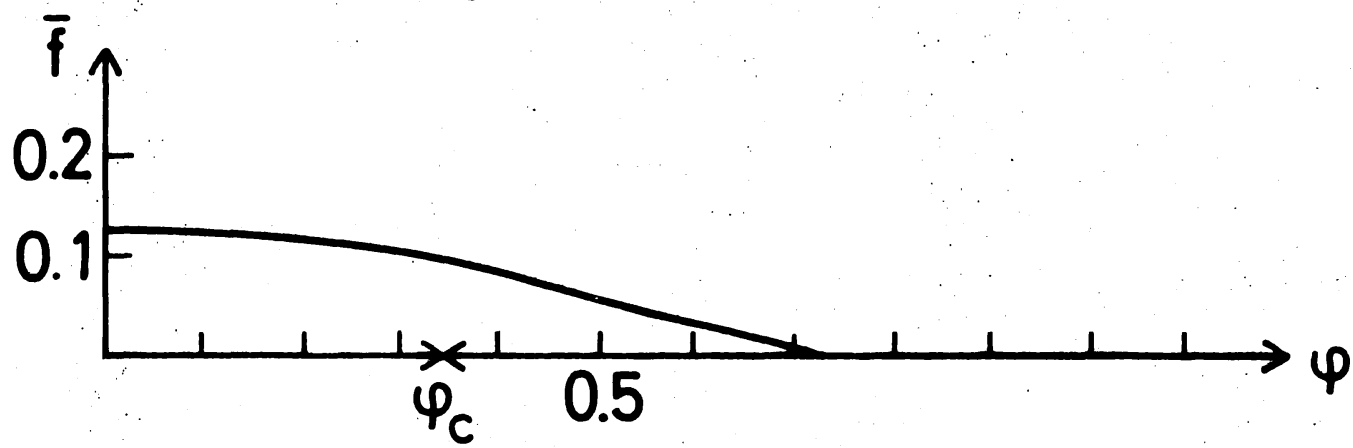

(v)

$A=2.0$

$B=1.0$

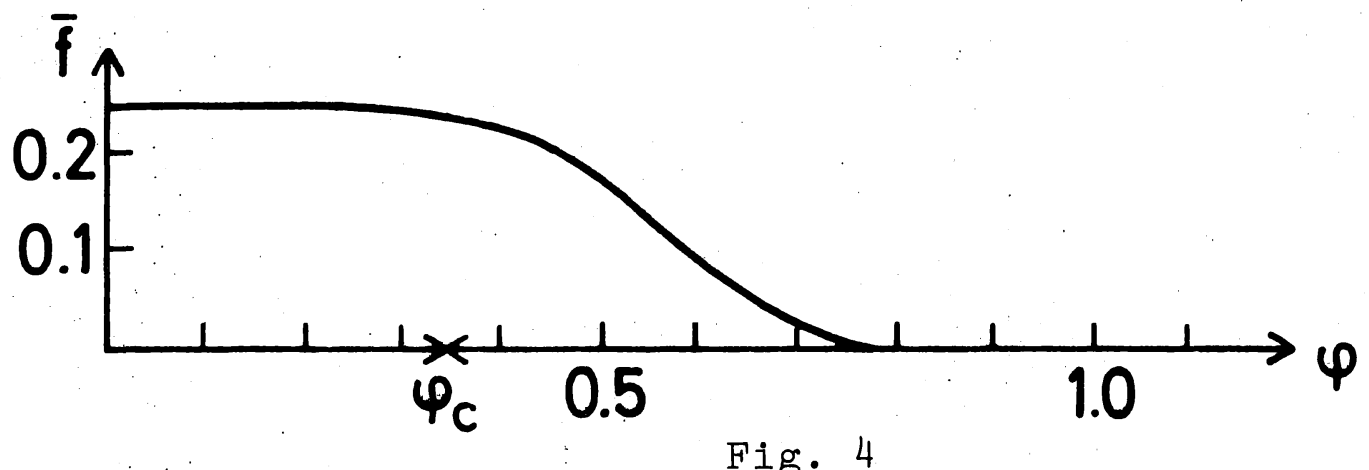

(vi)

$A=1.0$

$B=2.0$ 


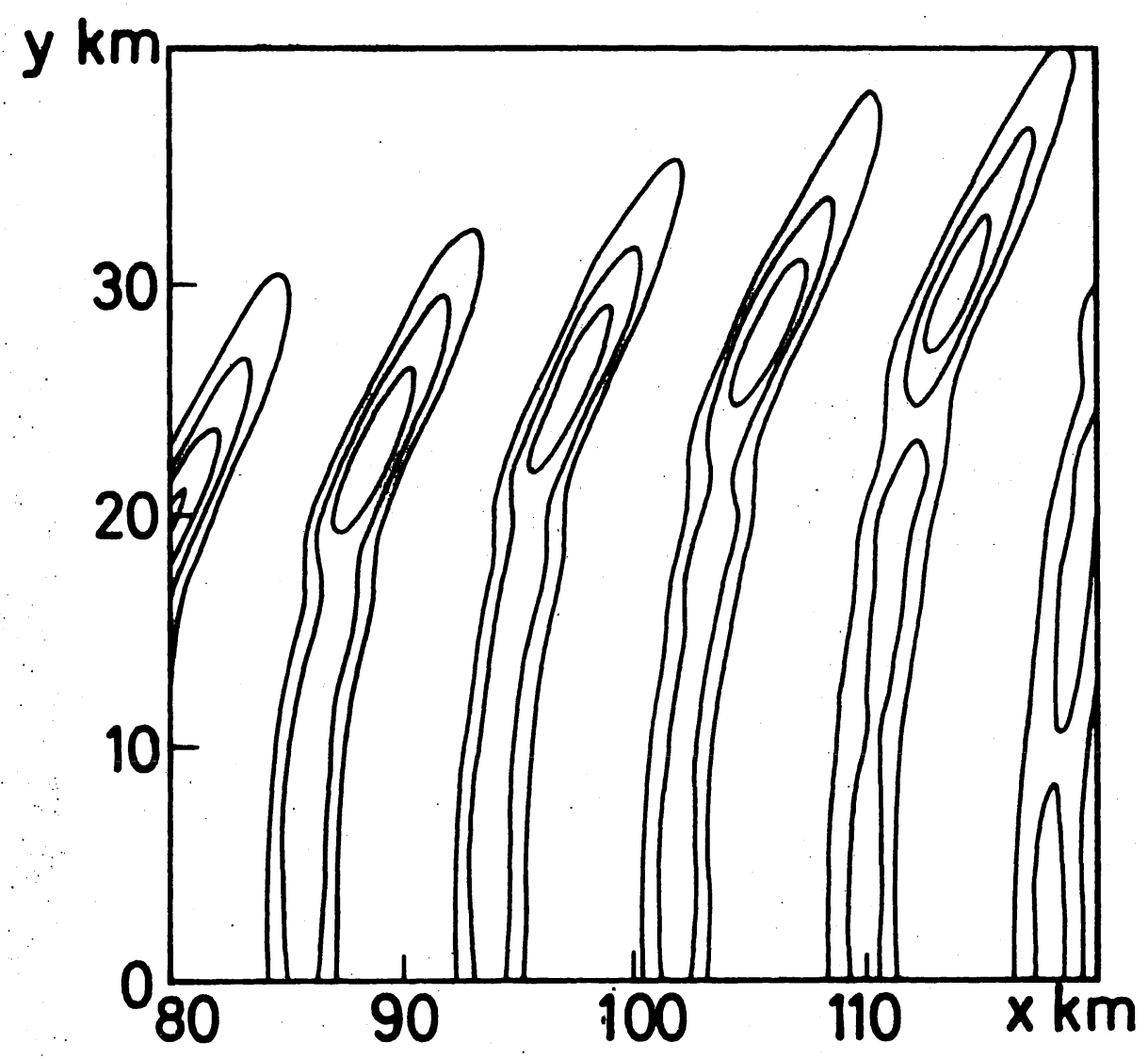

Fig. $5 a$

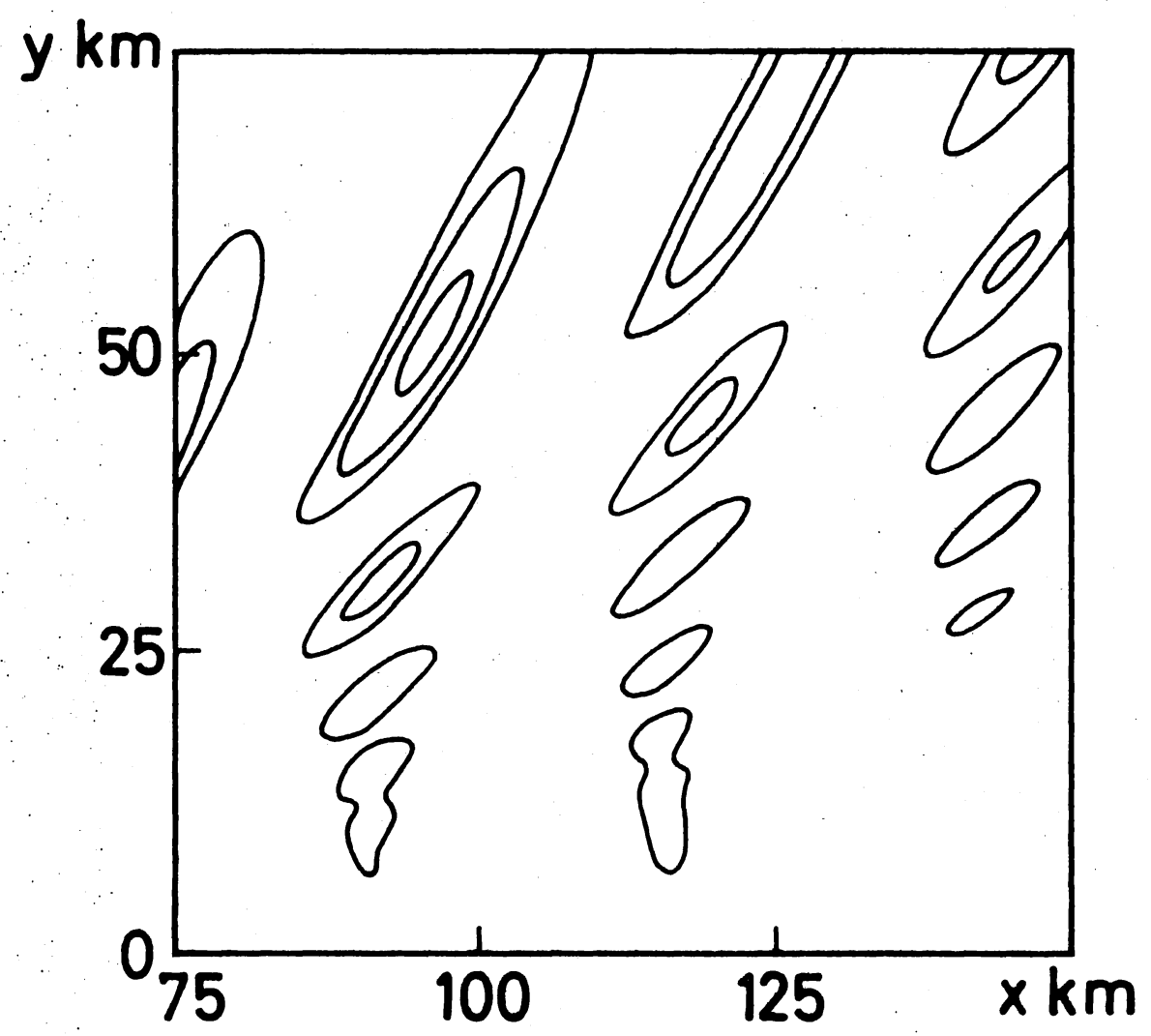

Fig. 5b 


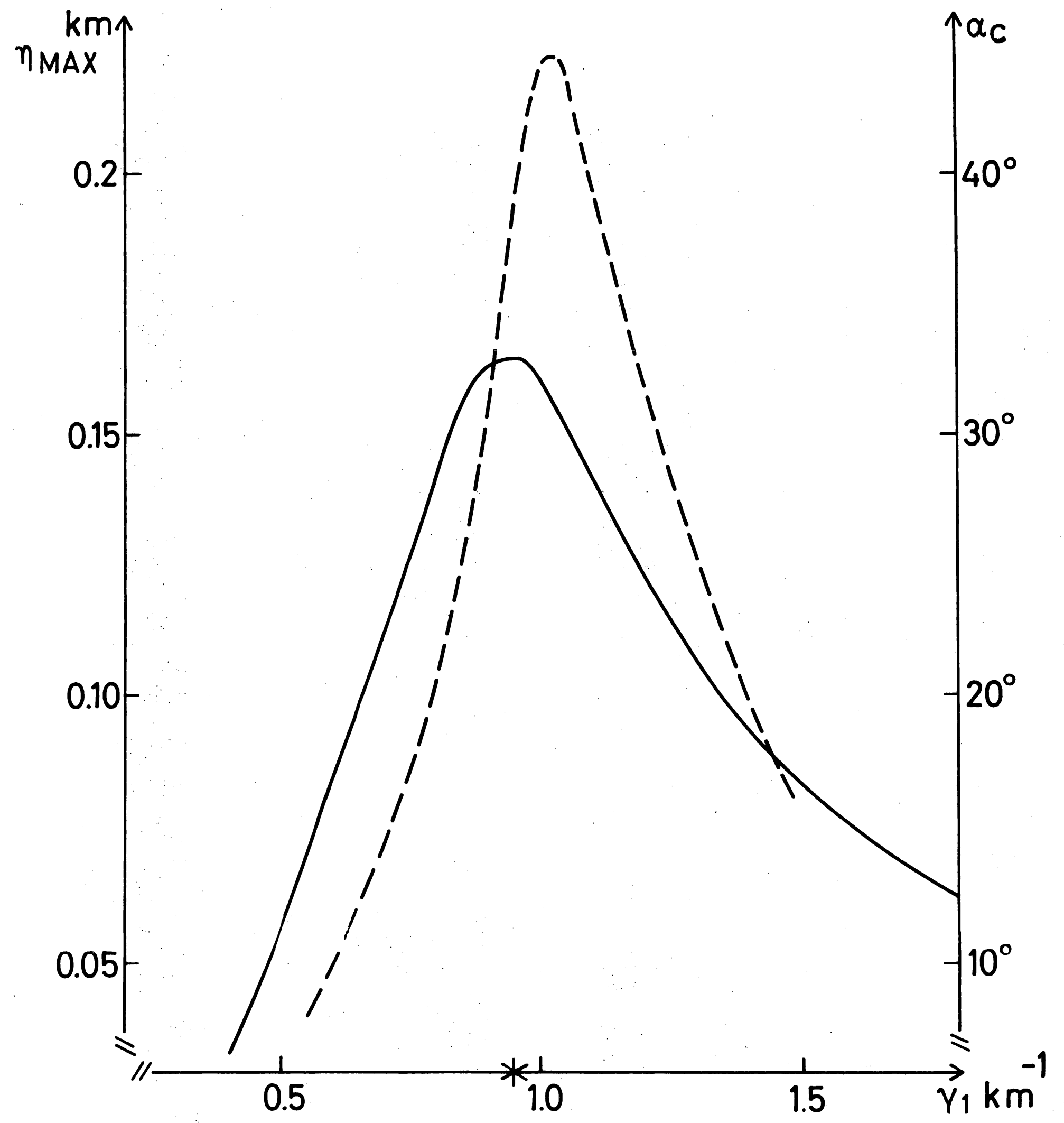

Fig. $6 a$ 


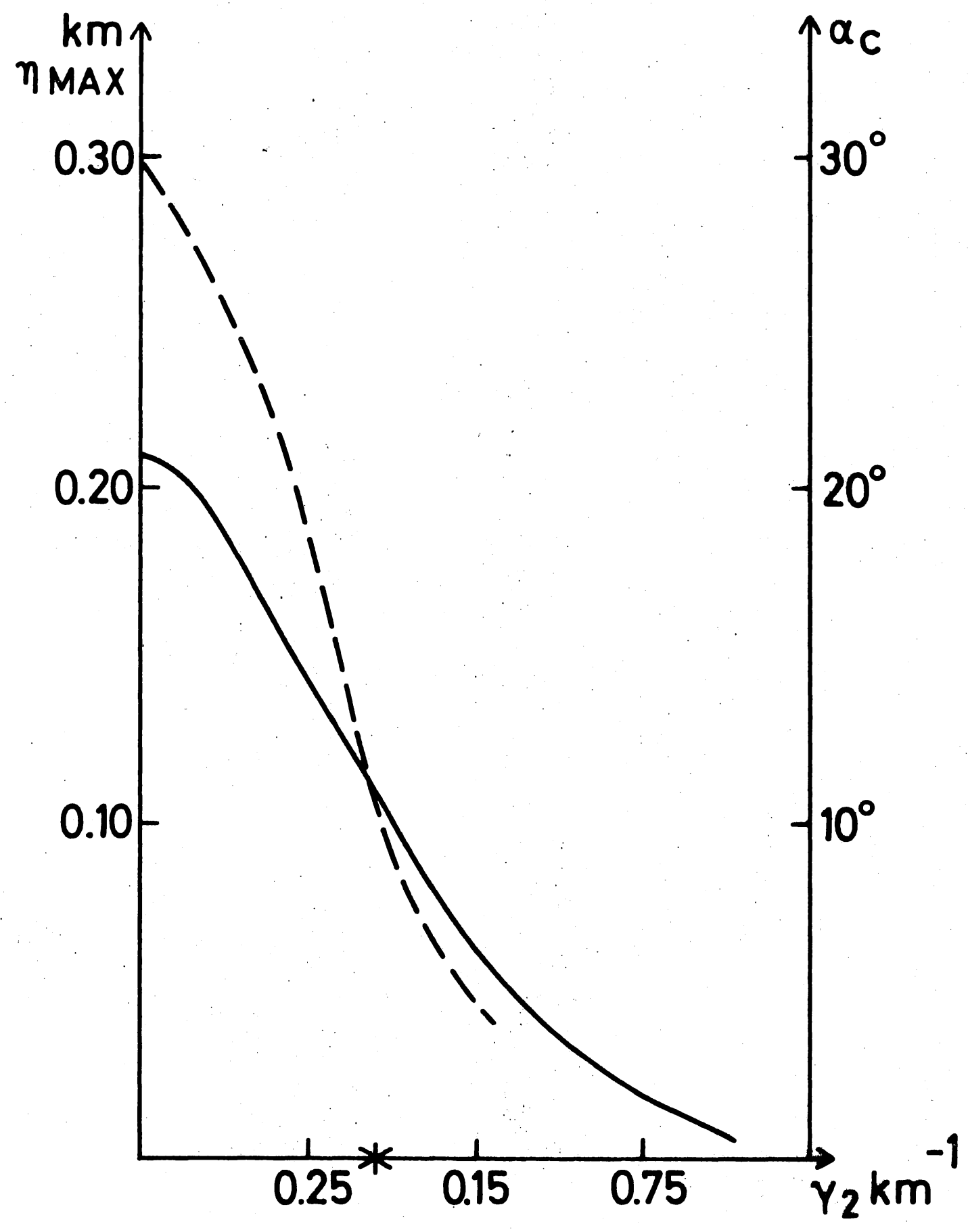

Fig. 6b 


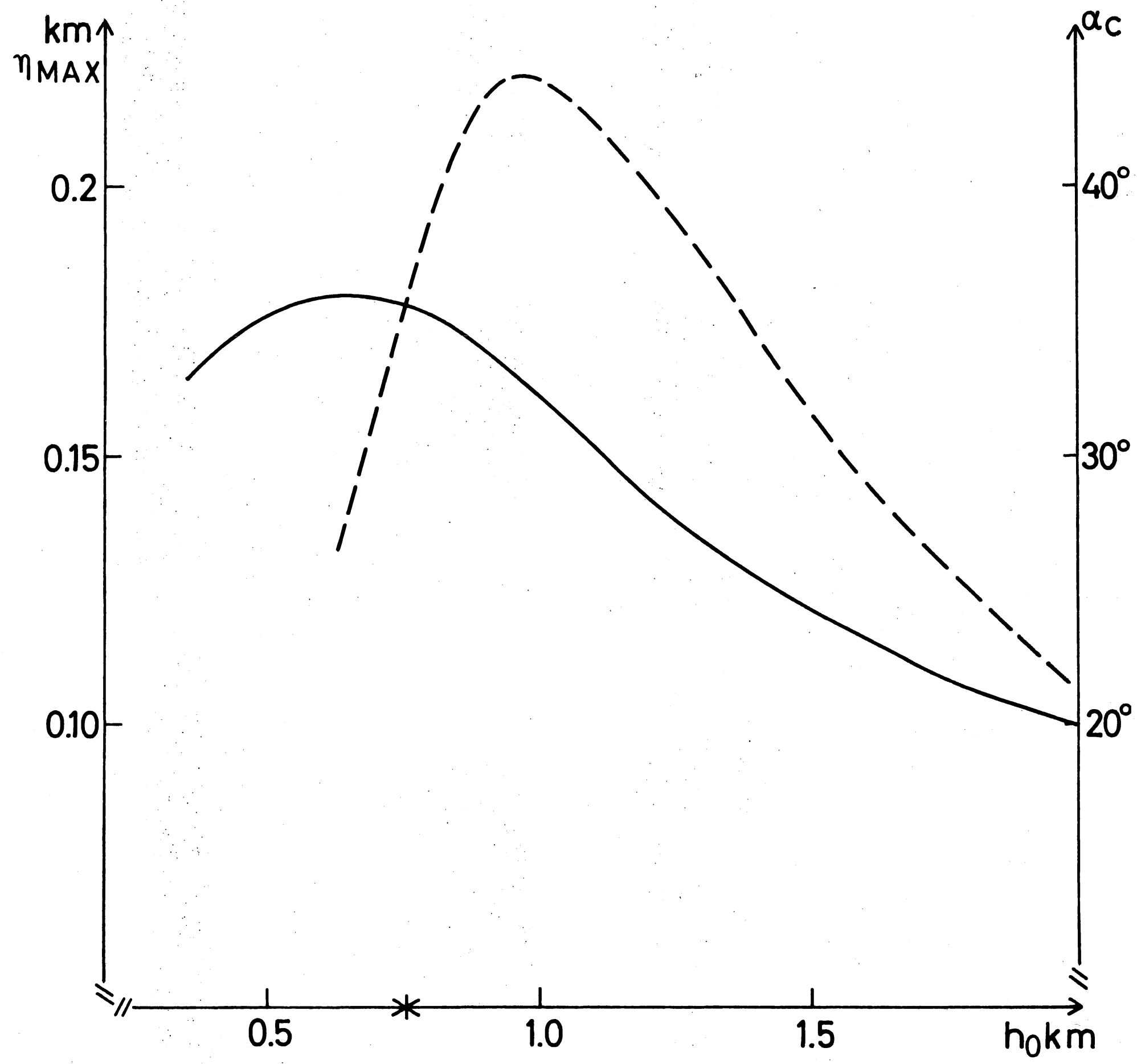

Fig. 6c 


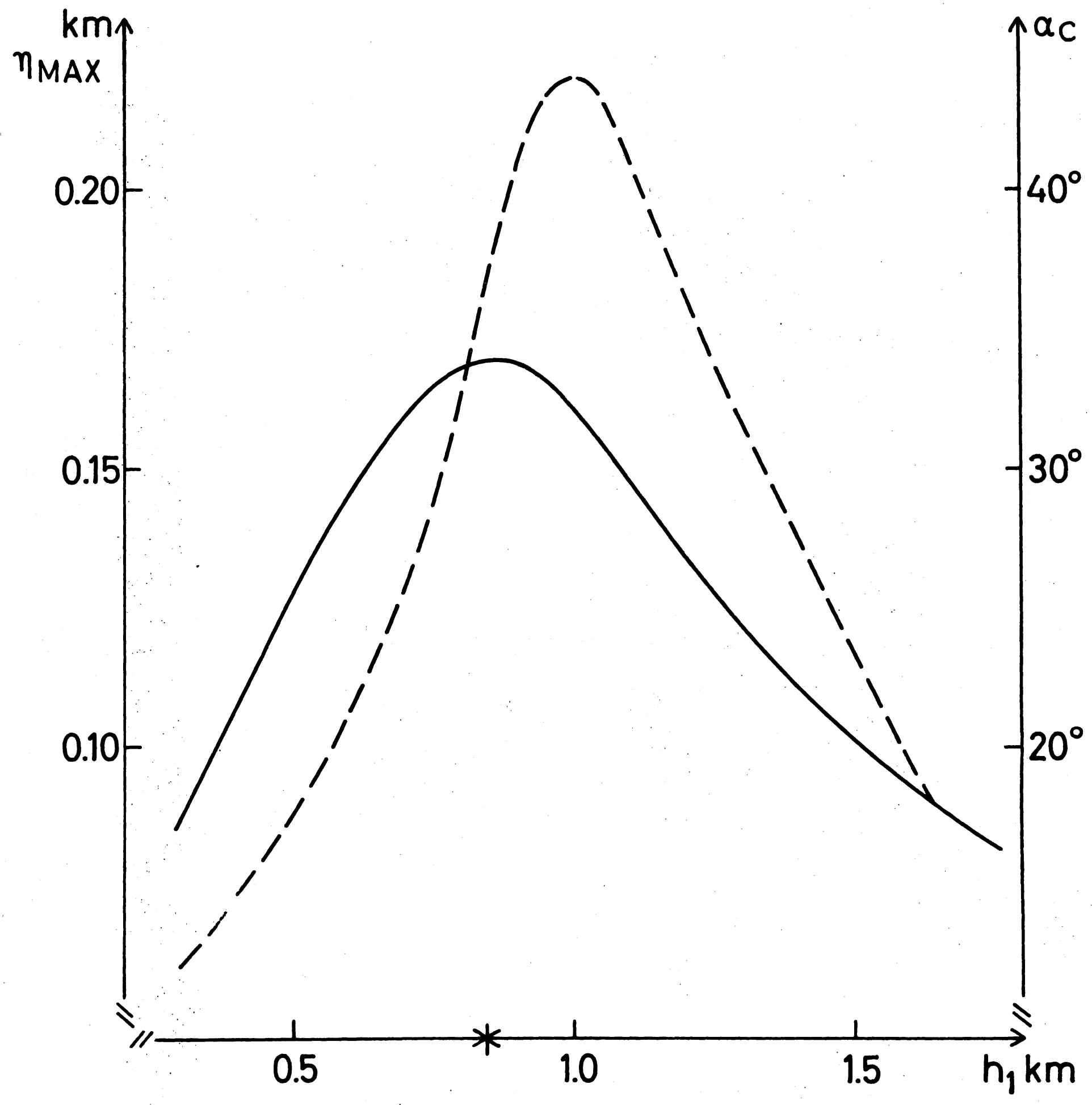

Fig. $6 d$ 


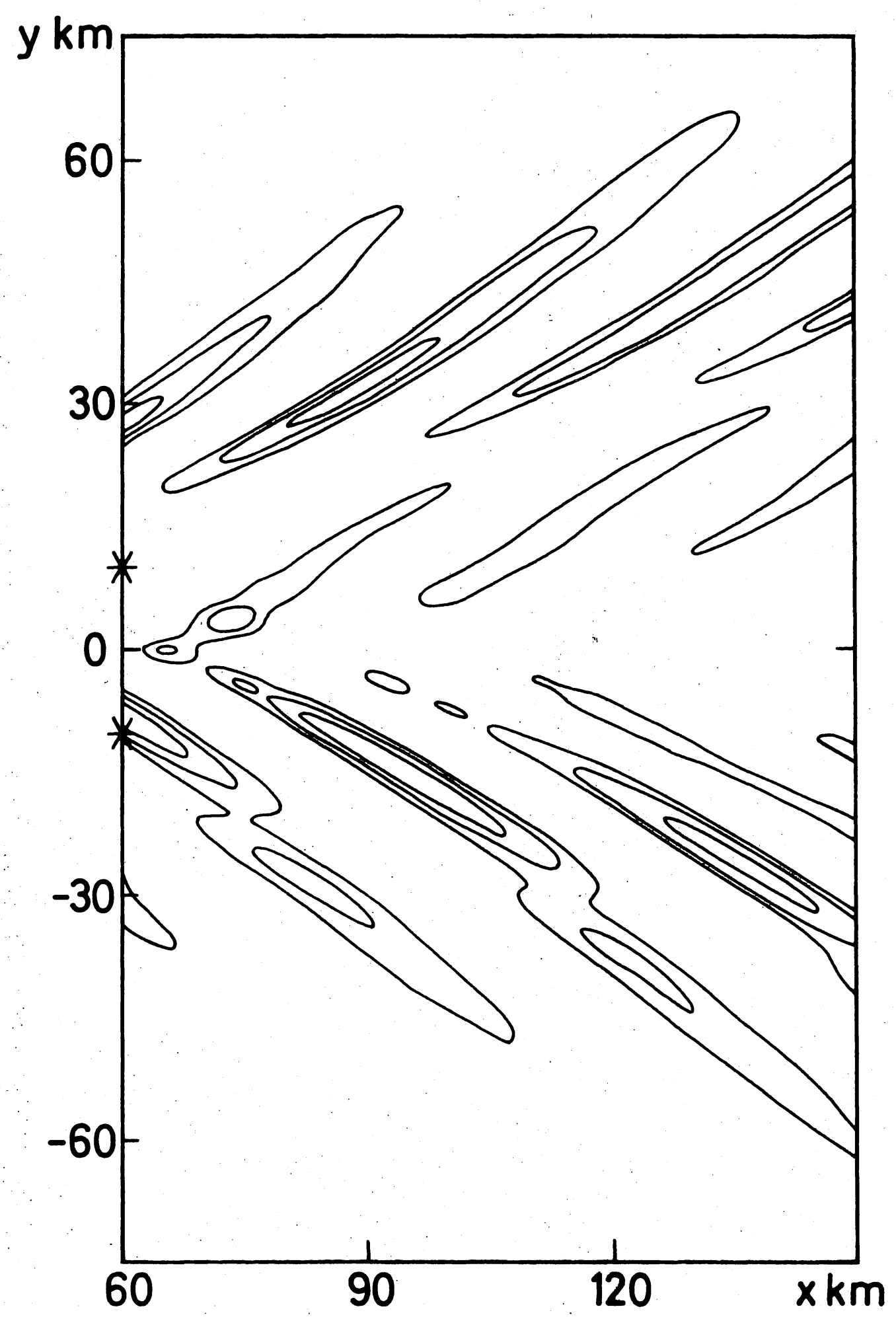

Fig. 7 


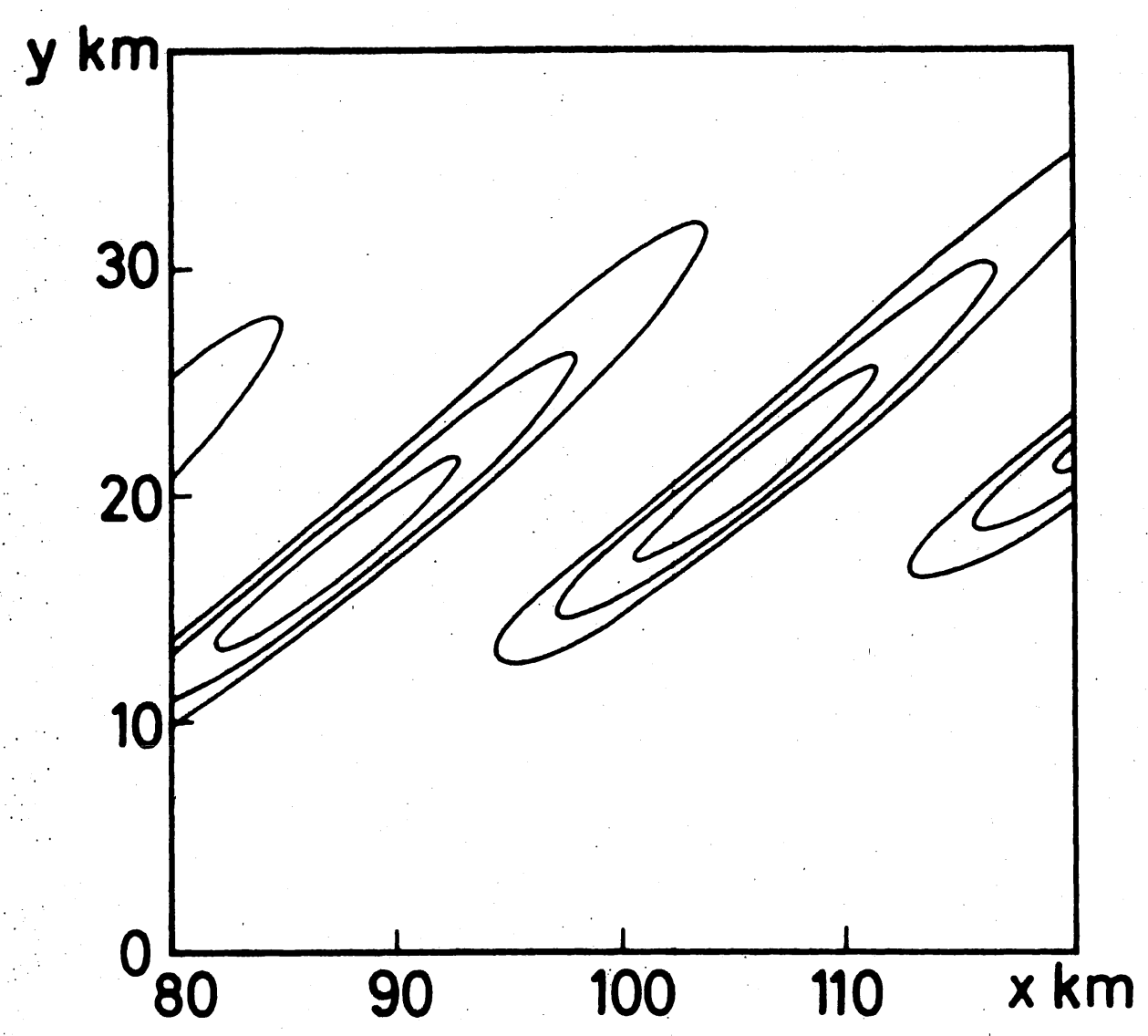

Fig. 8a

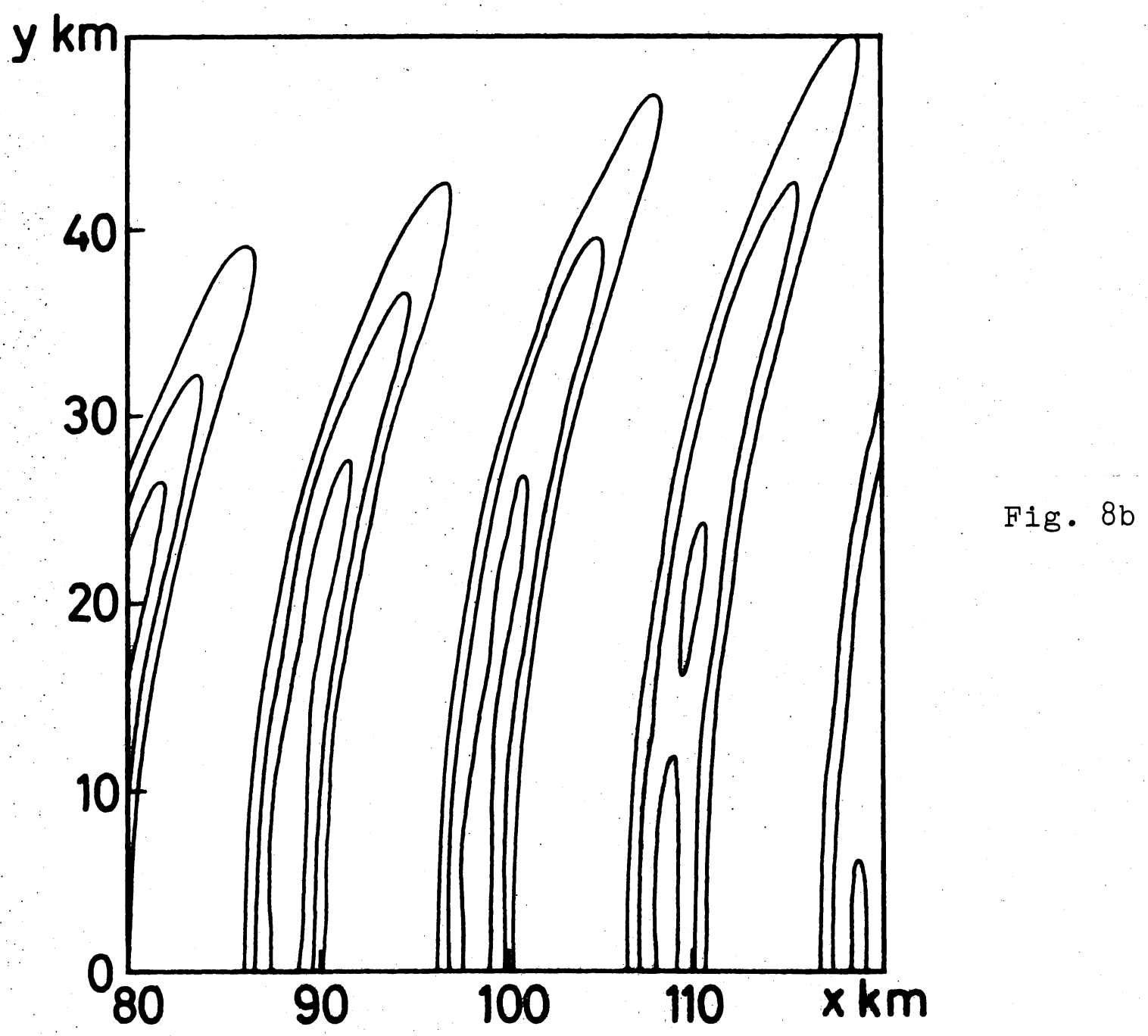

